Pacific Journal of Mathematics

ALGEBRAS AND FIBER BUNDLES

Michael GarDNer Fe 


\title{
ALGEBRAS AND FIBER BUNDLES
}

\author{
J. M. G. FELL
}

Let $A$ be an associative algebra and $\hat{A}_{n}$ the family of all equivalence classes of irreducible representations of $A$ of dimension exactly $n$. Topologizing $\hat{A}_{n}$ as in a paper about to appear in the Transactions of the American Mathematical Society, we show that for each $n, A$ gives rise to a fiber bundle having $\hat{A}_{n}$ as its base space and the $n \times n$ total matrix algebra as its fiber.

Throughout this note $A$ will be an arbitrary fixed associative algebra over the complex field $C$. By a representation of $A$ we understand a homomorphism $T$ of $A$ into the algebra of all linear endomorphisms of some complex linear space $H(T)$, the space of $T$. We write $\operatorname{dim}(T)$ for the dimension of $H(T)$. Irreducibility and equivalence of representations are understood in the purely algebraic sense. If $T$ is a representation, $r \cdot T$ will be the direct sum of $r$ copies of $T$. Let $\hat{A}^{(f)}$ the family of all equivalence classes of finitedimensional irreducible representations of $A$; and put

$$
\hat{A}^{(n)}=\left\{T \in \hat{A}^{(f)} \mid \operatorname{dim}(T) \leqq n\right\}, \hat{A}_{n}=\left\{T \in \hat{A}^{(f)} \mid \operatorname{dim}(T)=n\right\} .
$$

We shall usually not distinguish between representations and the equivalence classes to which they belong.

Let $T$ be a finite-dimensional representation of $A$. If for each $a$ in $A \tau(a)$ is the matrix of $T_{a}$ with respect to some fixed ordered basis of $H(T)$, then $\tau: a \rightarrow \tau(a)$ is a matrix representation of $A$ equivalent to $T$.

By $A^{\ddagger}$ we mean the space of all complex linear functionals on $A$, and by $\operatorname{Ker}(\varphi)$ the kernel of $\varphi$. If $T \in \hat{A}^{(f)}$, we put

$$
\Phi(T)=\left\{\varnothing \in A^{*} \mid \operatorname{Ker}(T) \subset \operatorname{Ker}(\varphi)\right\} .
$$

An element $\varphi$ of $A^{*}$ is associated with $T$ if $\varphi \in \Phi(T)$. One element of $\Phi(T)$ is of course the character $\chi^{T}$ of $T\left(\chi^{T}(a)=\right.$ Trace $\left(T_{a}\right)$ for $a$ in $A$ ). An element $T$ of $\hat{A}^{(f)}$ is uniquely determined by the knowledge of one nonzero functional in $\Phi(T)$ ([2], Proposition 2).

As in [2] we equip $\hat{A}^{(f)}$ with the functional topology as follows: If $T \in \hat{A}^{(f)}$ and $\mathscr{S} \subset \hat{A}^{(f)}, T$ belongs to the functional closure of $\mathscr{S}$ if $\Phi(T) \subset\left(\bigcup_{s \in \mathscr{S}} \Phi(S)\right)^{-}$where - denotes closure in the topology of pointwise convergence on $A$.

Our main object in this note is to prove the following fact about

Received November 11, 1964. 
the functional topology relativized to $\hat{A}_{n}$ :

THEOREM 1. Fix a positive integer $n$; and let $T$ be any element of $\hat{A}_{n}$. Then there exists a neighborhood $U$ of $T$ in $\hat{A}_{n}$, and a function $\tau$ assigning to each $S$ in $U$ a matrix representation $\tau_{S}$ of $A$ equivalent to $S$, such that for each $a$ in $A$ the matrix-valued function

$$
S \longrightarrow \tau_{S}(a)(S \in U)
$$

is continuous on $U$.

This asserts (see $\S 4$ ) that, for each $n, A$ gives rise to a fiber bundle with base space $\hat{A}_{n}$ whose fiber is the $n \times n$ total matrix algebra.

2. Preliminary results. The following Proposition 1 coincides with Proposition 7 of [2] (which was stated in [2] without proof). Proposition 1 is not required for what follows it; but its proof is related to later proofs.

Proposition 1. Let $n$ be a positive integer; and suppose that $\left\{T^{(i)}\right\}$ is a net of elements of $\hat{A}^{(n)}$ converging to each of the $p$ inequivalent elements $V^{1}, \cdots, V^{p}$ of $\hat{A}^{(n)}$. Then

$$
\sum_{s=1}^{p}\left(\operatorname{dim}\left(V^{s}\right)\right)^{2} \leqq n^{2}
$$

Proof. Let $m_{s}=\operatorname{dim}\left(V^{s}\right), q=\sum_{s=1}^{p} m_{s}^{2}$. Each $\Phi\left(V^{s}\right)$ has dimension $m_{\varepsilon}^{2}$, and by the Extended Burnside Theorem ([1], Theorem 27.8) the $\Phi\left(V^{s}\right)(s=1, \cdots, p)$ are linearly independent subspaces of $A^{\sharp}$. Thus there are $q$ linearly independent functionals $\varphi_{1}, \cdots, \varphi_{q}$ each of which is associated with some $V^{s}$. By the definition of the functional topology we can replace $\left\{T^{(i)}\right\}$ by a subnet, and choose for each $r=1, \cdots, q$ and each $i$ a functional $\varphi_{r}^{i}$ in $\Phi\left(T^{(i)}\right)$, such that

$$
\varphi_{r}^{i} \longrightarrow i \varphi_{r}(r=1, \cdots, q) \text {. }
$$

Since the $\varphi_{1}, \cdots, \varphi_{q}$ are independent, (2) implies that for some $i$ the $\varphi_{1}^{i}, \cdots, \varphi_{q}^{i}$ are independent. Since $\operatorname{dim}\left(\Phi\left(T^{(i)}\right)\right) \leqq n^{2}$, it follows that $q \leqq n^{2}$. This proves (1).

REMARK. If $A$ is a Banach algebra we have shown elsewhere ([2], Proposition 13) that a stronger inequality than (1) holds, namely

$$
\sum_{s=1}^{p} \operatorname{dim}\left(V^{s}\right) \leqq n
$$


Probably (3) holds for arbitrary $A$, but we have not been able to prove it.

CoRollaRy 1. $\hat{A}_{n}$ is Hausdorff for each $n$.

For each $\varphi$ in $A^{\prime}$ let us define $S^{\varphi}$ to be the natural representation of $A$ acting in $A / J$, where $J$ is the left ideal of $A$ consisting of those $a$ such that $\varphi(b a)=0$ for all $b$ in $A$.

Lemma 1. Let $\left\{\phi_{i}\right\}$ be a net of elements of $A^{*}$, converging pointwise to an element $\varphi$ of $A^{*}$; and suppose the $S^{\varphi}, S^{\varphi_{i}}$ are all finite-dimensional. Then

$$
\operatorname{dim}\left(S^{\varphi}\right) \leqq \lim _{i} \inf \operatorname{dim}\left(S^{\varphi_{i}}\right) .
$$

Further, if $\sigma$ is a matrix representation of $A$ equivalent to $S^{\varphi}$, there exists for each $i$ a matrix representation $\sigma^{i}$ of $A$ equivalent to $S^{\varphi_{i}}$ such that

$$
\lim _{i}\left(\sigma^{i}(a)\right)_{j k}=(\sigma(a))_{j k}
$$

for all $a$ in $A$ and all $j, k=1, \cdots, \operatorname{dim}\left(S^{\varphi}\right)$.

Proof. Let $\pi$ be the natural map of $A$ onto $A / J$, where $J=$ $\{a \in A \mid \varphi(b a)=0$ for all $b$ in $A\}$; and put $m=\operatorname{dim}\left(S^{\varphi}\right)$. Every element of $(A / J)^{\ddagger}$ is of the form

$$
\pi(a) \longrightarrow \varphi(b a) \quad(a \in A)
$$

for some $b$ in $A$. Hence there are elements $a_{1}, \cdots, a_{m} b_{1}, \cdots, b_{m}$ of $A$ satisfying

$$
\varphi\left(b, a_{k}\right)=\hat{o}_{j k}(j, k=1, \cdots, m) .
$$

Since $\varphi_{i} \rightarrow \varphi$, (6) implies that

$$
\operatorname{det}\left\{\left(\varphi_{i}\left(b_{j} a_{k}\right)\right)_{j, k=1, \ldots, m}\right\} \neq 0,
$$

and hence $\operatorname{dim}\left(S^{\varphi_{i}}\right) \geqq m$, for all large $i$. This proves (4).

Now the $a_{k}, b_{j}$ could have been chosen to satisfy not only (6) but also

$$
(\sigma(x))_{j k}=\varphi\left(b_{\jmath} x a_{k}\right)
$$

$(x \in A ; j, k=1, \cdots, m)$; assume this done. By (7), for each large $i$ there are unique complex numbers $c_{j k}^{i}(j, k=1, \cdots, m)$ such that the elements $b_{j}^{i}=\sum_{k=1}^{m} c_{j k}^{i} b_{k}$ satisfy 


$$
\varphi_{i}\left(b_{j}^{i} a_{k}\right)=\delta_{j k} \quad(j, k=1, \cdots, m)
$$

By (6) and (9)

$$
\lim _{i} c_{j k}^{i}=\delta_{j k}
$$

In view of (4) and (9), there are elements $a_{m+1}^{i}, \cdots, a_{p_{i}}^{i}, b_{m+1}^{i}, \cdots, b_{p_{i}}^{i}$ of $A$ (where $p_{i}=\operatorname{dim}\left(S^{\varphi_{i}}\right)$ ), such that

$$
\varphi_{i}\left(b_{j}^{i} a_{k}^{i}\right)=\delta_{j k}
$$

for all large $i$ and all $j, k=1, \cdots, p_{i}$; (here we agree that $a_{j}^{i}=a_{j}$ for $j=1, \cdots, m)$. Now, if $j, k=1, \cdots, p_{i}$ and $x \in A$, define

$$
\left(\sigma^{i}(x)\right)_{j k}=\varphi_{i}\left(b_{j}^{i} x a_{k}^{i}\right) \text {. }
$$

From (8), (10), and (11), we verify that $\sigma^{i}$ is a matrix representation equivalent to $S^{\varphi_{i}}$ and that (5) holds. This completes the proof.

The following corollary was stated without proof as Proposition 8 of [2].

CoRollary 2. For each positive integer $n$, the map $T \rightarrow \chi^{T}\left(T \in \hat{A}_{n}\right)$ is a homeomorphism of $\hat{A}_{n}$ into $A^{\sharp}$ (the latter having the topology of pointwise convergence on $A$ ).

Proof. Obviously $\chi^{T} \rightarrow T$ is continuous. To prove that $T \rightarrow \chi^{T}$ is continuous, we shall suppose that $T,\left\{T^{i}\right\}$ are elements of $\hat{A}_{n}$ and that $\varphi_{i} \longrightarrow i \chi^{T}$ pointwise on $A$, where for each $i \varphi_{i}$ is associated with $T^{i}$; and we shall prove that $\chi^{T^{i}} \longrightarrow i \chi^{T}$ pointwise on $A$. Clearly this is sufficient.

By [2], Proposition 1, $S^{x^{T}} \cong n \cdot T$ and $S^{\varphi_{i}} \cong r_{i} \cdot T^{i}$, where $r_{i} \leqq n$. By (4) $r_{i}=n$ for all large $i$. Hence by $(5) \chi^{T}(a)=1 / n$ Trace $\left(S_{a}^{\varphi}\right)=$ $\lim _{i} 1 / n$ Trace $\left(S_{a}^{\varphi_{i}}\right)=\lim _{i} \chi^{T^{i}}(a)$ for all $a$ in $A$. So $\chi^{T^{i}} \rightarrow \chi^{T}$, and the corollary is proved.

If $M$ is any finite-dimensional complex linear space, the family $\mathscr{F}$ of all linear subspaces of $M$ of fixed dimension $r(r \leqq \operatorname{dim}(M))$ has a natural compact topology. Indeed, if $G$ is the unitary group on $M$ (with respect to some fixed inner product), and $G_{0}$ is the subgroup of $G$ which leaves stable some fixed $L$ in $\mathscr{F}$, then $\mathscr{F}$ is in one-toone correspondence with $G / G_{0}$, and the (compact) topology of $\mathscr{F}$ which makes this correspondence a homeomorphism is independent of the inner product and of $L$.

If $p$ is any positive integer, $M_{p}$ will be the $p \times p$ total matrix algebra over the complexes. Fix a positive integer $n$; and let $\mathscr{L}^{P}$ be the family of all those subalgebras $A$ of $M_{n}$ s which contain 1 and are 
isomorphic with $M_{n}$. For each $A$ in $\mathscr{L}$ let $A^{\prime}$ be the commuting algebra of $A$ in $M_{n^{2}}$ :

$$
A^{\prime}=\left\{a \in M_{n^{2}} \mid a b=b a \text { for all } b \text { in } A\right\} .
$$

It is well known that $A^{\prime} \in \mathscr{L}$ and that $A^{\prime \prime}=A$ whenever $A \in \mathscr{L}$.

Lemma 2. The map $A \rightarrow A^{\prime}$ is continuous on $\mathscr{L}$ to $\mathscr{L}$ (with the topology discussed above).

Proof. If not, then, by the compactness of the space $\mathscr{M}$ of all $n^{2}$-dimensional subspaces of $M_{n^{2}}$, one can find a net $\left\{A_{i}\right\}$ of elements of $\mathscr{L}$ such that $A_{i} \rightarrow A, A_{i}^{\prime} \rightarrow B$, where $A \in \mathscr{L}, B \in \mathscr{l}, A^{\prime} \neq B$. Choose an element $b$ of $B$ which is not in $A^{\prime}$, and let $a$ be any element of $A$. Then for each $i$ we can choose an $a_{i}$ in $A_{i}$ and $b_{i}$ in $A_{i}^{\prime}$ so that $a_{i} \rightarrow a, b_{i} \rightarrow b$. Since $a_{i} b_{i}=b_{i} a_{i}$, passing to the limit we obtain $a b=b a$, whence $b \in A^{\prime}$, a contradiction.

Lemma 3. Let $A$ be in $\mathscr{L}$, and let $e$ be a minimal nonzero idempotent in $A$. Then there is a neighborhood $U$ of $A$ in $\mathscr{L}$, and a continuous function $w$ on $U$ to $M_{n^{2}}$ such that

(i) $w(A)=e$, and

(ii) for each $B$ in $U w(B)$ is a minimal nonzero idempotent in $B$.

Proof. Choose an element $a$ of $A$ whose spectrum in $A$ is $\{1,2, \cdots, n\}$, and such that the spectral idempotent (in $A$ ) corresponding to the eigenvalue 1 of $a$ is precisely $e$; that is,

$$
e=((n-1) !)^{-1}(2-a)(3-a) \cdots(n-a) .
$$

Introducing a Hilbert space inner product into $M_{n^{2}}$ in an arbitrary manner and projecting, we can construct a continuous function $\alpha$ on $\mathscr{L}$ to $M_{n^{2}}$ such that $\alpha(A)=a$ and $\alpha(B) \in B$ for each $B$ in $\mathscr{L}$. Let $\sigma(B)$ be the spectrum of $\alpha(B)$ (considered as an element either of $B$ or of $M_{n^{2}}$ ). Since $\alpha$ is continuous, $\sigma(B)$ is continuous as a function of $B$. Thus there is a neighborhood $U$ of $A$ in $\mathscr{L}$, and $n$ continuous complex functions $\lambda_{1}, \cdots, \lambda_{n}$ on $U$ such that

(i) $\lambda_{r}(A)=r(r=1, \cdots, n)$,

(ii) for each $B$ in $U$ the $\lambda_{1}(B), \cdots, \lambda_{n}(B)$ are all distinct, and

(iii) $\sigma(B)=\left\{\lambda_{1}(B), \cdots, \lambda_{n}(B)\right\}$ for each $B$ in $U$. Now, for $B$ in $U$, put

$$
\left.w(B)=\prod_{j=2}^{n}\left(\lambda_{j}(B)-\lambda_{1}(B)\right)^{-1}\left(\lambda_{j}(B) \cdot 1-\alpha(B)\right)\right) .
$$

Clearly $w$ is continuous on $U, w(B) \in B$ for each $B$ in $U$, and $w(A)=e$. 
Since $w(B)$ is the spectral idempotent corresponding to the eigenvalue $\lambda_{1}(B)$ of $\alpha(B)$ (which has multiplicity 1$), w(B)$ is a minimal idempotent of $B$ for each $B$ in $U$.

Lemma 4. If $A \in \mathscr{L}$, there is a neighborhood $U$ of $A$ in $\mathscr{L}$, and a continuous function $w$ on $U$ to $M_{n^{2}}$, such that, for each $B$ in $U, w(B)$ is a minimal idempotent of the commuting algebra of $B$.

Proof. This follows immediately from Lemmas 2 and 3.

3. Proof of Theorem 1. We have seen ([2], Proposition 1) that $S^{x^{T}} \cong n \cdot T$. Thus, putting $m=n^{2}$, we may choose elements $a_{1}, \cdots, a_{m}$, $b_{1}, \cdots, b_{m}$ of $A$ as in the proof of Lemma 1 so that

$$
\chi^{T}\left(b_{j} a_{k}\right)=\delta_{j k}(j, k=1, \cdots, m) .
$$

Since $S \rightarrow \chi^{S}$ is continuous on $\hat{A}_{n}$ (Corollary 2), there is a neighborhood $U^{\prime}$ of $T$ in $\hat{A}_{n}$ such that $\operatorname{det}\left(\chi^{S}\left(b_{j} a_{k}\right)\right)_{j, k} \neq 0$ for $S$ in $U^{\prime}$. Thus, as in the proof of Lemma 1 , for each $S$ in $U^{\prime}$ we find unique complex numbers $c_{j k}(S)$ such that the elements $b_{j}(S)=\sum_{k=1}^{m} c_{j k}(S) b_{k}$ satisfy

$$
\chi^{S}\left(b_{j}(S) a_{k}\right)=\delta_{j k}
$$

$\left(j, k=1, \cdots, m ; S \in U^{\prime}\right)$. We now set

$$
\left(\sigma_{S}(x)\right)_{j k}=\chi^{S}\left(b_{j}(S) x a_{k}\right)
$$

$\left(j, k=1, \cdots, m ; S \in U^{\prime} ; x \in A\right)$, and verify as in the proof of Lemma 1 that, for $S$ in $U^{\prime}, \sigma_{S}$ is a matrix representation of $A$ equivalent to $n \cdot S$. Since $S \rightarrow \chi^{S}$ is continuous (Corollary 2), the $c_{j k}(S)$ are continuous in $S$ on $U^{\prime}$, and so

$$
S \longrightarrow \sigma_{S}(x) \text { is continuous on } U^{\prime}
$$

for each $x$ in $A$.

Since $\sigma_{s} \cong n \cdot S$, Burnside's Theorem asserts that the range $\sigma_{S}(A)$ of $\sigma_{S}$ belongs to $\mathscr{L}$. Further, it follows from (14) that $S \rightarrow \sigma_{S}(A)$ is continuous on $U^{\prime}$ (in the topology of $n^{2}$-dimensional subspaces discussed in $\S 2$ ). Thus, by Lemma 4 , there is a neighborhood $U^{\prime \prime}$ of $T$ contained in $U^{\prime}$, and a function $w$ on $U^{\prime \prime}$ to $M_{m}$ such that, for each $S$ in $U^{\prime \prime}, w(S)$ is a minimal idempotent of the commuting algebra of $\sigma_{S}(A)$.

We now consider $M_{m}$ is acting on $C^{m}$ (the space of complex $m$ tuples). Let $v_{1}, \cdots, v_{m}$ be a basis of $C^{m}$ such that $v_{1}, \cdots, v_{n}$ is a basis of range $(w(T))$. By the continuity of $w$ there will be a neighborhood $U$ of $T$ contained in $U^{\prime \prime}$ such that

$$
w(S) v_{1}, \cdots, w(S) v_{n}, v_{n+1}, \cdots, v_{m}
$$


is a basis of $C^{m}$ for each $S$ in $U$ (the first $n$ vectors of (15) being, of course, a basis of range $(w(S)))$. Now for each $S$ in $U$ and $x$ in $A$ let $\rho_{S}(x)$ be the matrix of $\sigma_{S}(x)$ with respect to the ordered basis (15), and let $\tau_{S}(x)$ be the $n \times n$ matrix consisting of the first $n$ rows and columns of $\rho_{S}(x)$. Since $w(S)$ is a minimal idempotent of the commuting algebra of $\sigma_{S}(A), \sigma_{S}$ restricted to range $(w(S))$ is an irreducible subrepresentation of $\sigma_{S}$ and so is equivalent to $S$. Thus, for each $S$ in $U, \tau_{S}$ is a matrix representation of $A$ equivalent to $S$. Further, since $S \rightarrow w(S)$ is continuous on $U$, the basis (15) varies continuously with $S$ on $U$; and therefore by (14) we conclude that $S \rightarrow \tau_{S}(x)$ is continuous on $U$ for each $x$ in $A$. This completes the proof of Theorem 1.

4. Fiber bundles associated with $\mathrm{A}$. Fix a positive integer $n$, and let $G_{n}$ be the group of all algebraic automorphisms of the total matrix algebra $M_{n}$. We are going to describe to within equivalence a fiber bundle $B_{n}$ with base space $\hat{A}_{n}$, fiber $M_{n}$, and group $G_{n}$. To do so, it is sufficient to specify an open covering of $\hat{A}_{n}$, and to define on the overlap of any two sets in the covering the $G_{n}$-valued "coordinate transformation functions" ([3], §§2,3). As our open covering we take the set of all the $U=U_{r}\left(T \in \widehat{A}_{n}\right)$ of Theorem 1 . If $T, T^{\prime} \in \widehat{A}_{n}$, the coordinate transformation function $\Gamma_{T, T^{\prime}}$ on $U_{T} \cap U_{T^{\prime}}$ will assign to each $S$ in $U_{T} \cap U_{T}$, the following automorphism of $M_{n}$ :

$$
\Gamma_{T, T^{\prime}}(S): \tau_{S}^{\left(T^{\prime}\right)}(a) \longrightarrow \tau_{S}^{\left(T^{\prime \prime}\right)}(a) \quad(a \in A) .
$$

(Here $\tau^{(T)}$ is the $\tau$ of Theorem 1). The property $\Gamma_{T, T^{\prime \prime}}=\Gamma_{T, T^{\prime \prime}} \circ \Gamma_{T, T^{\prime}}$ (on $U_{T} \cap U_{T^{\prime}} \cap U_{T^{\prime \prime}}$ ) obviously holds; and the continuity of the maps $S \rightarrow \tau_{S}^{\left(T^{\prime}\right)}(a)$ and $S \rightarrow \tau_{S}^{\left(T^{\prime \prime}\right)}(a)$ assures us that $\Gamma_{r, T^{\prime}}$ is continuous. Thus we have defined a fiber bundle of the required kind; its equivalence class clearly depends only on $A$.

Thus, if the algebra $A$ has a large supply of finite-dimensional irreducible representations, the structure of the fiber bundles $B_{n}(n=$ $1,2, \cdots)$ constitutes a significant feature of the structure of $A$. We hope in a later paper to discuss the structure of these bundles for certain special kinds of algebras associated with locally compact groups having "large" compact subgroups.

\section{BIBLIOGRAPHY}

1. C. W. Curtis and I. Reiner, Representation Theory of Finite Groups and Associative Algebras, Interscience Publishers, 1962.

2. J. M. G. Fell, The dual spaces of Banach algebras, Trans. Amer. Math. Soc. 114 (1965), 227-250.

3. Norman Steenrod, The Topology of Fibre Bundles, Princeton University Press, 1951. 



\section{PACIFIC JOURNAL OF MATHEMATICS}

\section{EDITORS}

\author{
H. SAMmLSON \\ Stanford University \\ Stanford, California \\ R. M. BLUMENTHAL \\ University of Washington \\ Seattle, Washington 98105
}

\author{
*J. DUGUNDJI \\ University of Southern California \\ Los Angeles, California 90007
}

RICHARD ARENS

University of California

Los Angeles, California 90024

\section{E. F. BECKENBACH \\ B. H. NEUMANN \\ ASSOCIATE EDITORS}

\section{SUPPORTING INSTITUTIONS}

\author{
UNIVERSITY OF BRITISH COLUMBIA \\ CALIFORNIA INSTITUTE OF TECHNOLOGY \\ UNIVERSITY OF CALIFORNIA \\ MONTANA STATE UNIVERSITY \\ UNIVERSITY OF NEVADA \\ NEW MEXICO STATE UNIVERSITY \\ OREGON STATE UNIVERSITY \\ UNIVERSITY OF OREGON \\ OSAKA UNIVERSITY \\ UNIVERSITY OF SOUTHERN CALIFORNIA
}

\author{
STANFORD UNIVERSITY \\ UNIVERSITY OF TOKYO \\ UNIVERSITY OF UTAH \\ WASHINGTON STATE UNIVERSITY \\ UNIVERSITY OF WASHINGTON \\ * * * \\ AMERICAN MATHEMATICAL SOCIETY \\ CHEVRON RESEARCH CORPORATION \\ TRW SYSTEMS \\ NAVAL ORDNANCE TEST STATION
}

\footnotetext{
Mathematical papers intended for publication in the Pacific Journal of Mathematics should be typewritten (double spaced). The first paragraph or two must be capable of being used separately as a synopsis of the entire paper. It should not contain references to the bibliography. Manu. scripts may be sent to any one of the four editors. All other communications to the editors should be addressed to the managing editor, Richard Arens at the University of California, Los Angeles, California 90024.

50 reprints per author of each article are furnished free of charge; additional copies may be obtained at cost in multiples of 50 .
}

The Pacific Journal of Mathematics is published monthly. Effective with Volume 16 the price per volume (3 numbers) is $\$ 8.00$; single issues, $\$ 3.00$. Special price for current issues to individual faculty members of supporting institutions and to individual members of the American Mathematical Society: $\$ 4.00$ per volume; single issues $\$ 1.50$. Back numbers are available.

Subscriptions, orders for back numbers, and changes of address should be sent to Pacific Journal of Mathematics, 103 Highland Boulevard, Berkeley 8, California.

Printed at Kokusai Bunken Insatsusha (International Academic Printing Co., Ltd.), No. 6, 2-chome, Fujimi-cho, Chiyoda-ku, Tokyo, Japan.

PUBLISHED BY PACIFIC JOURNAL OF MATHEMATICS, A NON-PROFIT CORPORATION

The Supporting Institutions listed above contribute to the cost of publication of this Journal, but they are not owners or publishers and have no responsibility for its content or policies.

* Paul A. White, Acting Editor until J. Dugundji returns. 


\section{Pacific Journal of Mathematics}

\section{Vol. 16, No. $3 \quad$ BadMonth, 1966}

Gert Einar Torsten Almkvist, Stability of linear differential equations with

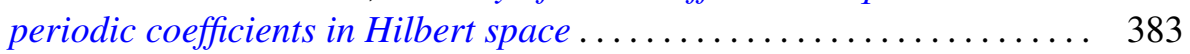

Richard Allen Askey and Stephen Wainger, A transplantation theorem for ultraspherical coefficients ................................ 393

Joseph Barback, Two notes on regressive isols .................. 407

Allen Richard Bernstein and Abraham Robinson, Solution of an invariant subspace problem of K. T. Smith and P. R. Halmos .............. 421

P. R. Halmos, Invariant subspaces of polynomially compact operators . . . . 433

Leon Bernstein, New infinite classes of periodic Jacobi-Perron algorithms.................................... 439

Richard Anthony Brualdi, Permanent of the direct product of matrices .... . 471

W. Wistar (William) Comfort and Kenneth Allen Ross, Pseudocompactness and uniform continuity in topological groups .................. 483

James Michael Gardner Fell, Algebras and fiber bundles . . . . . . . . . . . . 497

Alessandro Figà-Talamanca and Daniel Rider, A theorem of Littlewood and lacunary series for compact groups ..................... 505

David London, Two inequalities in nonnegative symmetric matrices...... 515

Norman Jay Pullman, Infinite products of substochastic matrices ........ 537

James McLean Sloss, Reflection and approximation by interpolation along

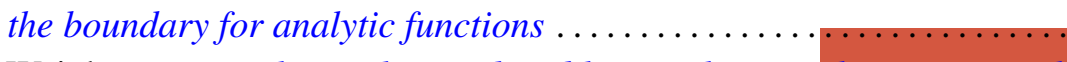

Carl Weinbaum, Visualizing the word problem, with an application to sixth groups................................... 